\title{
Stochastic Analysis of a Repairable System of Non- identical Units with Priority for Operation and Repair Subject to Weather Conditions
}

\author{
S.C Malik \\ Department of Statistics, \\ M.D. University, Rohtak-124001, \\ Haryana (India)
}

\author{
Savita Deswal \\ UGC Teacher Fellow \\ Department of Statistics, \\ M.D. University, Rohtak Haryana (India)
}

\begin{abstract}
A repairable system of two non-identical units - one is an original (called main unit) and other is a substandard (called duplicate unit) is investigated stochastically under different weather conditions - normal and abnormal. The system starts its operation with original unit keeping duplicate unit in cold standby. There is a direct failure of each unit from normal mode. Both units are capable in performing the system functions well. A single server is called immediately to do repair of the unit whenever needed. The operation and repair of the units are stopped in abnormal weather. And, in normal weather, priority is given for operation and repair to the original unit over duplicate unit. After repair unit works as new. All random variables are statistically independent and uncorrelated. The failure time of the units and time to change of weather conditions follow negative exponential distributions while repair times of the units are arbitrarily distributed. Various reliability and performance measures are obtained in steady state using semi-Markov process and regenerative point technique. The graphical behavior of MTSF, availability and profit function with respect to normal weather rate has also been observed for a particular case.
\end{abstract}

\section{KEYWORDS}

Repairable system, Non-identical units, Different weather conditions, Priority, Reliability measures and Stochastic analysis.

\section{INTRODUCTION}

It is a common knowledge that redundancy can be used to improve the performance and reliability of repairable systems. Therefore, stochastic models of cold standby repairable systems with identical units have widely been studied by the researchers including Naidu and Gopalan [1] , Goyal and Sharma [2] and Singh [3] under strict control of weather conditions. In fact, it is very difficult to afford an identical unit in spare in case its cost is high. In such a situation, a substandard unit might be kept as spare in cold standby not only to improve the reliability but also to protect the operation of the system for a considerable time. Mokkadis et al. [4] analyzed stochastically the redundant systems of non-identical units under different sets of assumption on failure and repair. Also, sometimes it becomes necessary to give priority in operation and repair to one unit over the other in order to make the system more profitable. A good example of the situation is that of a system consisting of one unit as power supply through electric transformer and other unit generator. The priority is obviously given to the power supply coming through electric transformer rather than generator. The generator will be used only when supply through electric transformer is discontinued. Further, due to costly operation of generator, the priority for repair may be given to transformer over generator. Chander [5] discussed reliability models with priority for operation and repair.

Further, the weather conditions cannot be controlled easily by human beings. The conditions may change due to changing climate, earthquake, vibrations and other unforeseen reasons. Therefore, Malik and Barak [6] evaluated reliability and economic measures of a single -unit system with no operation and repair in abnormal weather. It is also pointed out here that not much work related to stochastic analysis of repairable systems of non-identical units with priority for operation and repair subject to different weather conditions has been reported so far in the literature of reliability.

In view of the above, the present paper deals with the stochastic analysis of a repairable system of two non-identical units - one is original (called main unit) and other is substandard (called a duplicate unit). The system performs under two weather conditions - normal and abnormal. Initially, the main unit is operative and duplicate unit is kept as spare in cold standby. Each unit has direct complete failure from normal mode. Both units are capable of performing the system functions well with different degree of reliability and desirability. There is a single server who visits the system immediately whenever needed. The operation and repair of the units are stopped in abnormal weather. And, in normal weather, priority is given for operation and repair of the main unit over duplicate unit. After repair, each unit works as new. All random variables are statistically independent and uncorrelated. The switch devices are perfect. The failure times of the units and time of change of weather conditions follow negative exponential distributions. And, repair times of the units are arbitrarily distributed. Various reliability and performance measures such as transition probabilities, mean sojourn times, mean time to system failure (MTSF), steady state availability, busy period of the server, expected no of visits by the server and profit function are obtained using semi-Markov process and regenerative point technique. The graphical behavior of MTSF, availability and profit functions with respect to normal weather rate has also been examined for a particular case. 


\section{NOTATIONS}

$\mathrm{E}$

MO/DO

: The set of regenerative states

$\overline{\text { MWO }}$,

$\overline{\mathrm{DWO}} \quad$ : Main/Duplicate unit is good but waiting for operation due to abnormal weather

DCs : Duplicate unit is in cold standby mode

$\lambda / \lambda 1 \quad$ : Constant failure rate of Original /Duplicate unit

$\beta / \beta 1 \quad$ : Constant rate of change of weather from normal to abnormal/abnormal to normal weather

MFur/DFur : Main/duplicate unit failed and under repair

MFUR/DFUR: Main/duplicate unit failed and under repair continuously from previous state

MFwr/DFwr : Main/duplicate unit failed and waiting for repair

MFWR/DFWR : Main/duplicate unit failed and waiting for repair continuously from previous state

\section{$\overline{\mathrm{MFwr}}$ /}

$\overline{\mathrm{DFwr}}$ : Main/Duplicate unit failed and waiting for repair due to abnormal weather

\section{$\overline{\text { MFWR }}$ /}

$\overline{\text { DFWR }}:$ Main/Duplicate unit failed and waiting for repair continuously from previous state due to abnormal weather

$\mathrm{g}(\mathrm{t}) / \mathrm{G}(\mathrm{t}) \quad$ : pdf/cdf of repair time of Original unit g1 (t) / G1(t) : pdf/cdf of repair time of Duplicate unit qij (t) / Qij (t) : pdf/cdf of passage time from regenerative state $\mathrm{i}$ to a regenerative state $\mathrm{j}$ or to a failed state $\mathrm{j}$ without visiting any other regenerative state in $(0, \mathrm{t}]$

qij.kr (t) /

Qij.kr (t) : pdf/cdf of direct transition time from Regenerative state $\mathrm{i}$ to a regenerative state $\mathrm{j}$ or to a failed state $\mathrm{j}$ visiting state $\mathrm{k}, \mathrm{r}$ once in $(0, \mathrm{t}]$

q ij.k,(r,s)n(t)

/Qij.k,(r,s)n(t) : pdf/cdf of direct transition time from regenerative state $i$ to a regenerative state $\mathrm{j}$ or to a failed state $\mathrm{j}$ visiting state $\mathrm{k}$ once and $\mathrm{n}$ times states $\mathrm{r}$ and $\mathrm{s}$.
Mi(t) : Probability that the system is up initially in regenerative state $\mathrm{Si}$ at time $\mathrm{t}$ without visiting to any other regenerative state

Wi(t) : Probability that the server is busy in state Si upto time $t$ without making any transition to any other regenerative state or returning to the same via one or more non-regenerative states

mij : The unconditional mean time taken by the system to transits from any regenerative state $\mathrm{Si} C \mathrm{~S}$ when time is counted from epoch of entrance into that state $\mathrm{Sj}$. Mathematically, it can be written as mij $=\int t Q i j(t)=-q i j * '(0)$

$\mu_{\mathrm{i}}$

: The mean sojourn time in state $\mathrm{Si}$ this is given by $\mu_{\mathrm{i}}=\mathrm{E}(\mathrm{t})=\int \mathrm{P}(\mathrm{T}>\mathrm{t}) \mathrm{dt}=\sum \mathrm{jmij}$, where $\mathrm{T}$ denotes the time to system failure

(S)/@/@n : Symbol for Laplace Stieltjes Convolution / Laplace convolution / Laplace convolution $\mathrm{n}$ times

$\sim$ / : Symbol for Laplace Steiltjes Transform (LST)/ Laplace Transform (LT)

' (desh) : Used to represent derivative

The following are the possible transition states of the system $\mathrm{S} 0=(\mathrm{MO}, \mathrm{DCs}), \mathrm{S} 1=$ (MFur, DO),

$\mathrm{S} 2=(\overline{\mathrm{MWO}}, \overline{\mathrm{DCs}}), \mathrm{S} 3=(\overline{\mathrm{MFwr}}, \overline{\mathrm{DWO}})$,

S4 = (MFUR, DFwr), S5 = (MO, DFur $)$

$\mathrm{S} 6=(\overline{\mathrm{MFwr}}, \overline{\mathrm{DFWR}}), \mathrm{S} 7=(\mathrm{MFur}, \mathrm{DFWR})$,

$\mathrm{S} 8=(\overline{\mathrm{MWO}}, \overline{\mathrm{DFwr}}), \mathrm{S} 9=(\mathrm{MFur}, \mathrm{DFwr})$

The states S0, S1, S2, S3, S5, S8, S9 are regenerative while the states $\mathrm{S} 4, \mathrm{~S} 6, \mathrm{~S} 7$ are non regenerative as shown in figure1. 


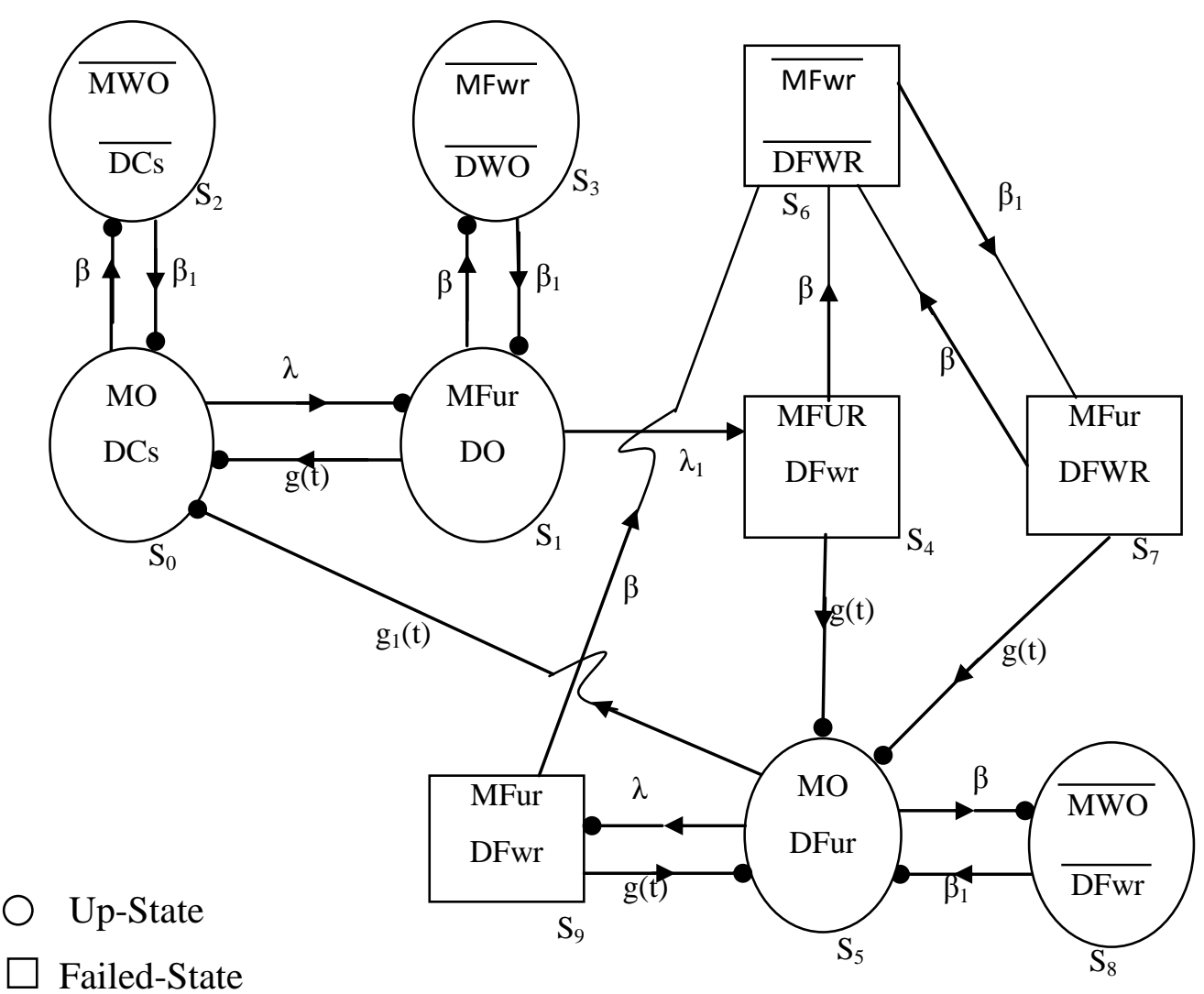

- Regenerative point

Fig. 1 State Transition Diagram

\section{RELIABILITY INDICES}

\subsection{Transition Probabilities and Mean} Sojourn Times

Simple probabilistic considerations yield the following expressions for the non-zero elements $\mathrm{p}_{\mathrm{ij}}=\mathrm{Q}_{\mathrm{ij}}(\infty)=\int \mathrm{q}_{\mathrm{ij}}(\mathrm{t}) \mathrm{dt}$ we have

$\mathrm{p}_{01}=\frac{\lambda}{\beta+\lambda}, \mathrm{p}_{02}=\frac{\beta}{\beta+\lambda}, \mathrm{p}_{10}=\mathrm{g} *\left(\beta+\lambda_{1}\right)$,

$\mathrm{p}_{13}=\frac{\beta}{\beta+\lambda_{1}}\left(1-\mathrm{g} *\left(\beta+\lambda_{1}\right), \mathrm{p}_{14}=\frac{\lambda_{1}}{\beta+\lambda_{1}}\left(1-\mathrm{g} *\left(\beta+\lambda_{1}\right), \mathrm{p}_{20}=1\right.\right.$,

$\mathrm{p}_{31}=1, \mathrm{p}_{45}=\mathrm{g} *(\beta), \mathrm{p}_{46}=1-\mathrm{g} *(\beta), \mathrm{p}_{50}=\mathrm{g}_{1} *(\beta+\lambda)$,

$\mathrm{p}_{58}=\frac{\beta}{\beta+\lambda}\left(1-\mathrm{g}_{1} *(\beta+\lambda)\right), \mathrm{p}_{59}=\frac{\lambda}{\beta+\lambda}\left(1-\mathrm{g}_{1} *(\beta+\lambda)\right)$,

$\mathrm{p}_{67}=1, \mathrm{p}_{75}=\mathrm{g} *(\beta), \mathrm{p}_{76}=1-\mathrm{g} *(\beta), \mathrm{p}_{85}=1, \mathrm{p}_{95}=\mathrm{g} *(\beta)$,

$\mathrm{p}_{96}=1-\mathrm{g} *(\beta)$
It can be easily verified that

$\mathrm{p}_{01}+\mathrm{p}_{02}=\mathrm{p}_{10}+\mathrm{p}_{13}+\mathrm{p}_{14}=\mathrm{p}_{20}=\mathrm{p}_{31}=\mathrm{p}_{45}+\mathrm{p}_{46}=$

$\mathrm{p}_{50}+\mathrm{p}_{58}+\mathrm{p}_{59}=\mathrm{p}_{67}=\mathrm{p}_{75}+\mathrm{p}_{76}=\mathrm{p}_{85}=\mathrm{p}_{95}+\mathrm{p}_{96}=1$

The mean sojourn times $\left(\mu_{\mathrm{i}}\right)$ in the state $S_{\mathrm{i}}$ are

$\mu_{0}=\frac{1}{\beta+\lambda}, \mu_{1}=\frac{1}{\beta+\lambda_{1}}\left(1-\mathrm{g}^{*}\left(\beta+\lambda_{1}\right)\right)$,

$\mu_{2}=\frac{1}{\beta_{1}}, \mu_{3}=\frac{1}{\beta_{1}}, \mu_{4}=\frac{1}{\beta}\left(1-\mathrm{g}^{*}(\beta)\right.$,

$\mu_{5}=\frac{1}{\beta+\lambda}\left(1-\mathrm{g}_{1} *(\beta+\lambda)\right), \mu_{6}=\frac{1}{\beta_{1}}$,

$\mu_{7}=\frac{1}{\beta}\left(1-g^{*}(\beta), \mu_{8}=\frac{1}{\beta_{1}}, \mu_{9}=\frac{1}{\beta}\left(1-g^{*}(\beta)\right)\right.$

Also 
$\mathrm{m}_{01}+\mathrm{m}_{02}=\mu_{0}, \mathrm{~m}_{10}+\mathrm{m}_{13}+\mathrm{m}_{14}=\mu_{1}, \mathrm{~m}_{20}=\mu_{2}, \mathrm{~m}_{31}=\mu_{3}$,

$\mathrm{m}_{45}+\mathrm{m}_{46}=\mu_{4}, \mathrm{~m}_{50}+\mathrm{m}_{58}+\mathrm{m}_{59}=\mu_{5}, \mathrm{~m}_{67}=\mu_{6}, \mathrm{~m}_{75}+\mathrm{m}_{76}=\mu_{7}$,

$\mathrm{m}_{85}=\mu_{8}, \mathrm{~m}_{95}+\mathrm{m}_{96}=\mu_{9}$

and

$\left.\mu_{1}^{\prime}=m_{10}+m_{13}+m_{15.4}+m_{15.4,(6,7}\right)^{n} \mu_{9}^{\prime}=m_{95}+m_{95 .(6,7)}{ }^{n}$

\subsection{Reliability and Mean Time to System Failure (MTSF)}

Let $\phi_{\mathrm{i}}(\mathrm{t})$ be the cdf of first passage time from regenerative state $\mathrm{i}$ to a failed state. Regarding the failed state as absorbing state, we have the following recursive relations for $\phi_{\mathrm{i}}(\mathrm{t})$ :

$\phi_{0}(\mathrm{t})=\mathrm{Q}_{01}(\mathrm{t}) \mathrm{S} \phi_{1}(\mathrm{t})+\mathrm{Q}_{02}(\mathrm{t}) \mathrm{S} \phi_{2}(\mathrm{t})$

$\phi_{1}(\mathrm{t})=\mathrm{Q}_{10}(\mathrm{t}) \mathrm{S} \phi_{0}(\mathrm{t})+\mathrm{Q}_{13}(\mathrm{t}) \mathrm{S} \phi_{1}(\mathrm{t})+\mathrm{Q}_{14}(\mathrm{t})$

$\phi_{2}(\mathrm{t})=\mathrm{Q}_{20}(\mathrm{t}) \mathrm{S} \phi_{0}(\mathrm{t}), \phi_{3}(\mathrm{t})=\mathrm{Q}_{31}(\mathrm{t}) \mathrm{S} \phi_{1}(\mathrm{t})$

Taking LST of above relation (6) and solving for $\tilde{\phi}_{0}(s)$

We have

$\mathrm{R} *(\mathrm{~s})=\frac{1-\tilde{\phi}_{0}(s)}{s}$

The reliability of the system model can be obtained by taking Laplace inverse transform of (7).

The mean time to system failure (MTSF) is given by

$\mathrm{MTSF}=\lim _{s \rightarrow o} \frac{1-\tilde{\phi}_{0}(s)}{s}=\frac{N_{1}}{D_{1}}$

where

$\mathrm{N}_{1}=\mathrm{p}_{01}\left(\mathrm{p}_{13} \mu_{3}+\mu_{1}\right)+\left(1-\mathrm{p}_{13}\right)\left(\mu_{0}+\mathrm{p}_{02} \mu_{2}\right)$

$\mathrm{D}_{1}=\mathrm{p}_{01} \mathrm{p}_{14}$

\subsection{Steady State Availability}

Let $A_{i}(t)$ be the probability that the system is in up-state at instant ' $t$ ' given that the system entered regenerative state $\mathrm{i}$ at $\mathrm{t}=0$.The recursive relations for $A_{i}(t)$ are given as

$\mathrm{A}_{0}(\mathrm{t})=\mathrm{M}_{0}(\mathrm{t})+\mathrm{q}_{01}(\mathrm{t})\left(\mathrm{A}_{1}(\mathrm{t})+\mathrm{q}_{02}(\mathrm{t}) \odot \mathrm{A}_{2}(\mathrm{t})\right.$

$\mathrm{A}_{1}(\mathrm{t})=\mathrm{M}_{1}(\mathrm{t})+\mathrm{q}_{10}(\mathrm{t}) \oplus \mathrm{A}_{0}(\mathrm{t})+\mathrm{q}_{13}(\mathrm{t}) \odot \mathrm{A}_{3}(\mathrm{t})$

$\left.+\left(\mathrm{q}_{15.4}(\mathrm{t})+\mathrm{q}_{15.4,(6,7)}\right)^{\mathrm{n}}(\mathrm{t})\right) \odot \mathrm{A}_{5}(\mathrm{t})$

$\mathrm{A}_{2}(\mathrm{t})=\mathrm{q}_{20}(\mathrm{t}) \odot \mathrm{A}_{0}(\mathrm{t}), \mathrm{A}_{3}(\mathrm{t})=\mathrm{q}_{31}(\mathrm{t}) \odot \mathrm{A}_{1}(\mathrm{t})$

$A_{5}(t)=M_{5}(t)+q_{50}(t) @ A_{0}(t)+q_{58}(t) \odot A_{8}(t)+q_{59}(t) @ A_{9}(t)$

$$
\mathrm{A}_{8}(\mathrm{t})=\mathrm{q}_{85}(\mathrm{t}) \oplus \mathrm{A}_{5}(\mathrm{t}), \mathrm{A}_{9}(\mathrm{t})=\left(\mathrm{q}_{95}(\mathrm{t})+\mathrm{q}_{95 .(6,7)}{ }^{\mathrm{n}}\right)(\mathrm{t}) \oplus \mathrm{A}_{5}(\mathrm{t})
$$

where $\mathrm{M}_{\mathrm{i}}(\mathrm{t})$ is the probability that the system is up initially in state $S_{i} \in E$ is up at time $t$ without visiting to any other regenerative state, we have

$M_{0}(t)=e^{-(\beta+\lambda) t}, M_{1}(t)=e^{-(\beta+\lambda) t} \overline{G(t)}, M_{5}(t)=e^{-\beta t} \overline{G(t)}(11)$

Taking LT of above relations (10) and solving for $\mathrm{A}_{0} *(\mathrm{~s})$.

The steady state availability is given by

$A_{0}(\infty)=\lim _{s \rightarrow 0} s A_{0}^{*}(s)=\frac{N_{2}}{D_{2}}$

where

$\mathrm{N}_{2}=\mathrm{p}_{50}\left(\mu_{0}\left(1-\mathrm{p}_{13}\right)+\mu_{1} \mathrm{p}_{01}\right)+\mu_{5} \mathrm{p}_{01} \mathrm{p}_{14}$

and

$\mathrm{D}_{2}=\mathrm{p}_{01} \mathrm{p}_{14}\left(\mu_{5}+\mu_{8} \mathrm{p}_{58}+\mathrm{p}_{59} \mu_{9}^{\prime}\right)+\mathrm{p}_{50}\left(\mathrm{p}_{01}\left(\mu_{1}^{\prime}+\mathrm{p}_{13} \mu_{3}\right)+\right.$

$\left.\left(1-\mathrm{p}_{13}\right)\left(\mu_{0}+\mathrm{p}_{02} \mu_{2}\right)\right)$

\subsection{Busy period analysis for server}

Let $B_{i}(t)$ be the probability that the server is busy in repairing the unit at an instant ' $t$ ' given that the system entered regenerative state $\mathrm{i}$ at $\mathrm{t}=0$. The recursive relations for $\mathrm{B}_{\mathrm{i}}(\mathrm{t})$ are as follows:

$\mathrm{B}_{0}(\mathrm{t})=\mathrm{q}_{01}(\mathrm{t}) \odot \mathrm{B}_{1}(\mathrm{t})+\mathrm{q}_{02}(\mathrm{t}) \odot \mathrm{B}_{2}(\mathrm{t})$

$\mathrm{B}_{1}(\mathrm{t})=\mathrm{W}_{1}(\mathrm{t})+\mathrm{q}_{10}(\mathrm{t}) \odot \mathrm{B}_{0}(\mathrm{t})+\mathrm{q}_{13}(\mathrm{t}) \odot \mathrm{B}_{3}(\mathrm{t})$

$$
\left.+\left(\mathrm{q}_{15.4}(\mathrm{t})+\mathrm{q}_{15.4,(6,7)}\right)^{\mathrm{n}}(\mathrm{t})\right) \odot \mathrm{B}_{5}(\mathrm{t})
$$

$B_{2}(t)=q_{20}(t) \odot B_{0}(t), B_{3}(t)=q_{31}(t) \odot B_{1}(t)$

$\mathrm{B}_{5}(\mathrm{t})=\mathrm{W}_{5}(\mathrm{t})+\mathrm{q}_{50}(\mathrm{t}) \oplus \mathrm{B}_{0}(\mathrm{t})+\mathrm{q}_{58}(\mathrm{t}) \oplus \mathrm{B}_{8}(\mathrm{t})+\mathrm{q}_{59}(\mathrm{t}) \oplus \mathrm{B}_{9}(\mathrm{t})$

$\mathrm{B}_{8}(\mathrm{t})=\mathrm{q}_{85}(\mathrm{t}) \odot \mathrm{B}_{5}(\mathrm{t})$,

$\mathrm{B}_{9}(\mathrm{t})=\mathrm{W}_{9}(\mathrm{t})+\left(\mathrm{q}_{95}(\mathrm{t})+\mathrm{q}_{95 .(6,7)}{ }^{\mathrm{n}}(\mathrm{t})\right) \propto \mathrm{B}_{5}(\mathrm{t})$

where $W_{i}(t)$ be the probability that the server is busy in state $S_{i}$ due to failure upto time $t$ without making any transition to any other regenerative state or returning to the same via one or more non regenerative states so,

$\mathrm{W}_{1}(\mathrm{t})=\mathrm{e}^{-(\beta+\lambda)}{ }_{1}^{\mathrm{t}} \overline{\mathrm{G}(\mathrm{t})}+\left(\lambda_{1} \mathrm{e}^{-(\beta+\lambda) \mathrm{t}} \odot 1\right) \overline{\mathrm{G}(\mathrm{t})}$,

$W_{5}(t)=e^{-(\beta+\lambda) t}, W_{9}(t)=e^{-\beta t} \overline{G(t)}$

Taking LT of above relations (14) and solving for $B_{0}^{*}(\mathrm{~s})$.The time for which server is busy due to repair is given by 


$$
\mathrm{B}_{0} *(\infty)=\lim _{s \rightarrow 0} s B_{0}^{*}(\mathrm{~s})=\frac{N_{3}}{D_{2}}
$$

where

$\mathrm{N}_{3}=\mathrm{p}_{01} \mathrm{p}_{50} \mathrm{~W}_{1} *(0)+\mathrm{p}_{01} \mathrm{p}_{14}\left(\mathrm{~W}_{5} *(0)+\mathrm{W}_{9} *(0) \mathrm{p}_{59}\right)$

and $\mathrm{D}_{2}$ is already mentioned.

\subsection{Expected number of visits by the server}

Let $N_{i}(t)$ be the expected number of visits by the server in $(0, t]$ given that the system entered the regenerative state $\mathrm{i}$ at $\mathrm{t}=0$. The recursive relations for $\mathrm{N}_{\mathrm{i}}(\mathrm{t})$ are given as

$\mathrm{N}_{0}(\mathrm{t})=\mathrm{Q}_{01}(\mathrm{t})(\mathrm{S})\left[1+\mathrm{N}_{1}(\mathrm{t})\right]+\mathrm{Q}_{02}(\mathrm{t})\left(\mathrm{S} \mathrm{N}_{2}(\mathrm{t})\right]$

$\mathrm{N}_{1}(\mathrm{t})=\mathrm{Q}_{10}(\mathrm{t})(\mathrm{S}) \mathrm{N}_{0}(\mathrm{t})+\mathrm{Q}_{13}(\mathrm{t})\left(\mathrm{S} \mathrm{N}_{3}(\mathrm{t})\right.$

$+\left(\mathrm{Q}_{15.4}(\mathrm{t})+\mathrm{Q}_{15.4,(67)}{ }^{\mathrm{n}}\right)\left(\mathrm{S} \mathrm{N}_{5}(\mathrm{t})\right.$

$\mathrm{N}_{2}(\mathrm{t})=\mathrm{Q}_{20}(\mathrm{t})\left(\mathrm{S} \mathrm{N}_{0}(\mathrm{t}), \mathrm{N}_{3}(\mathrm{t})=\mathrm{Q}_{31}(\mathrm{t}) \mathrm{SN}_{1}(\mathrm{t})\right.$

$\mathrm{N}_{5}(\mathrm{t})=\mathrm{Q}_{50}(\mathrm{t})(\mathrm{S}) \mathrm{N}_{0}(\mathrm{t})+\mathrm{Q}_{58}(\mathrm{t})\left(\mathrm{S} \mathrm{N}_{8}(\mathrm{t})+\mathrm{Q}_{59}(\mathrm{t})\left(\mathrm{S} \mathrm{N}_{9}(\mathrm{t})\right.\right.$

$\mathrm{N}_{8}(\mathrm{t})=\mathrm{Q}_{85}(\mathrm{t})\left(\mathrm{S} \mathrm{N}_{5}(\mathrm{t})\right.$,

$\left.\mathrm{N}_{9}(\mathrm{t})=\mathrm{Q}_{95}(\mathrm{t})+\mathrm{Q}_{95 .(6,7)}{ }^{\mathrm{n}}(\mathrm{t})\right)\left(\mathrm{S} \mathrm{N}_{5}(\mathrm{t})\right.$

Taking LST of relations (16) and solving for $\tilde{N}_{0}(s)$.

The expected numbers of visits per unit time by the server are given by

$N_{0}(\infty)=\lim _{s \rightarrow 0} s \tilde{N}_{0}(s)=\frac{N_{4}}{D_{2}}$

where

$\mathrm{N}_{4}=\mathrm{p}_{01} \mathrm{p}_{50}\left(1-\mathrm{p}_{13}\right)$

and $\mathrm{D}_{2}$ is already specified.

\subsection{Profit Analysis}

The profit incurred to the system model in steady state caN be obtained as

$\mathrm{P}_{\mathrm{i}}=\mathrm{K}_{0} \mathrm{~A}_{0}-\mathrm{K}_{1} \mathrm{~B}_{0}-\mathrm{K}_{2} \mathrm{~N}_{0}$

where

$\mathrm{K}_{0}=$ Revenue per unit up-time of the system

$\mathrm{K}_{1}=$ Cost per unit for which server is busy

$\mathrm{K}_{2}=$ Cost per unit visit by the server

and $\mathrm{A}_{0}, \mathrm{~B}_{0}, \mathrm{~N}_{0}$ are already defined.

\section{Particular Case}

Suppose $g(t)=\alpha \mathrm{e}^{-\alpha \mathrm{t}}, \mathrm{g}_{1}(\mathrm{t})=\alpha_{1} \mathrm{e}^{-\alpha}{ }_{1} \mathrm{t}$
By using the non zero elements $\mathrm{p}_{\mathrm{ij}}$, we can obtain the following results:

$\mathrm{p}_{01}=\frac{\lambda}{\beta+\lambda}, \mathrm{p}_{02}=\frac{\beta}{\beta+\lambda}, \mathrm{p}_{10}=\frac{\alpha}{\alpha+\beta+\lambda_{1}}, \mathrm{p}_{13}=\frac{\beta}{\alpha+\beta+\lambda_{1}}$,

$\mathrm{p}_{14}=\frac{\lambda_{1}}{\alpha+\beta+\lambda_{1}}, \mathrm{p}_{20}=1, \mathrm{p}_{31}=1, \mathrm{p}_{45}=\frac{\alpha}{\alpha+\beta}, \mathrm{p}_{46}=\frac{\beta}{\alpha+\beta}$,

$\mathrm{p}_{50}=\frac{\alpha_{1}}{\alpha_{1}+\beta+\lambda}, \mathrm{p}_{58}=\frac{\beta}{\alpha_{1}+\beta+\lambda}, \mathrm{p}_{59}=\frac{\lambda}{\alpha_{1}+\beta+\lambda}$,

$\mathrm{p}_{67}=1, \mathrm{p}_{75}=\frac{\alpha}{\alpha+\beta}, \mathrm{p}_{76}=\frac{\beta}{\alpha+\beta}, \mathrm{p}_{85}=1$,

$\mathrm{p}_{95}=\frac{\alpha}{\alpha+\beta}, \mathrm{p}_{96}=\frac{\beta}{\alpha+\beta}$

also

$\mu_{0}=\frac{1}{\beta+\lambda}, \mu_{1}=\frac{1}{\alpha+\beta+\lambda_{1}}, \mu_{2}=\frac{1}{\beta_{1}}, \mu_{3}=\frac{1}{\beta_{1}}, \mu_{4}=\frac{1}{\alpha+\beta}$,

$\mu_{5}=\frac{1}{\alpha_{1}+\beta+\lambda}, \mu_{6}=\frac{1}{\beta_{1}}, \mu_{7}=\frac{1}{\alpha+\beta}, \mu_{8}=\frac{1}{\beta_{1}}, \mu_{9}=\frac{1}{\alpha+\beta}$

$\mu_{1}^{\prime}=\frac{\alpha \beta_{1}\left(\alpha+\beta+\lambda_{1}\right)+\lambda_{1} \beta\left(\alpha+\beta+\beta_{1}\right)}{\alpha \beta_{1}\left(\alpha+\beta+\lambda_{1}\right)(\alpha+\beta)}, \mu_{9}^{\prime}=\frac{\left(\beta+\beta_{1}\right)}{\alpha \beta_{1}}$

$\operatorname{MTSF}\left(\mathrm{T}_{0}\right)=\frac{N_{1}}{D_{1}}$,

Steady state availability $\left(\mathrm{A}_{0}\right)=\frac{N_{2}}{D_{2}}$,

Busy period analysis for server $\left(\mathrm{B}_{0}\right)=\frac{N_{3}}{D_{2}}$,

Expected number of visits by the server $\left(\mathrm{N}_{0}\right)=\frac{N_{4}}{D_{2}}$

Where

$\mathrm{N}_{1}=\left(\alpha+\lambda+\lambda_{1}\right)\left(\beta+\beta_{1}\right)$

$\mathrm{D}_{1}=\lambda \lambda_{1} \beta_{1}$

$\mathrm{N}_{2}=\alpha \beta_{1}\left(\alpha_{1}(\alpha+\lambda)+\lambda_{1}\left(\alpha_{1}+\lambda\right)\right)$

$\mathrm{D}_{2}=\left(\beta+\beta_{1}\right)(\alpha+\lambda)\left(\lambda \lambda_{1}+\alpha_{1}\left(\alpha+\lambda_{1}\right)\right)$

$\mathrm{N}_{3}=\frac{\beta_{1} \lambda\left(\alpha_{1}\left(\alpha+\lambda_{1}\right)(\beta+\lambda)+\alpha \lambda_{1}(\alpha+\beta+\lambda)\right)}{(\alpha+\beta)}$

$\mathrm{N}_{4}=\alpha \beta_{1} \lambda \alpha_{1}\left(\alpha_{1}+\lambda\right)$ 


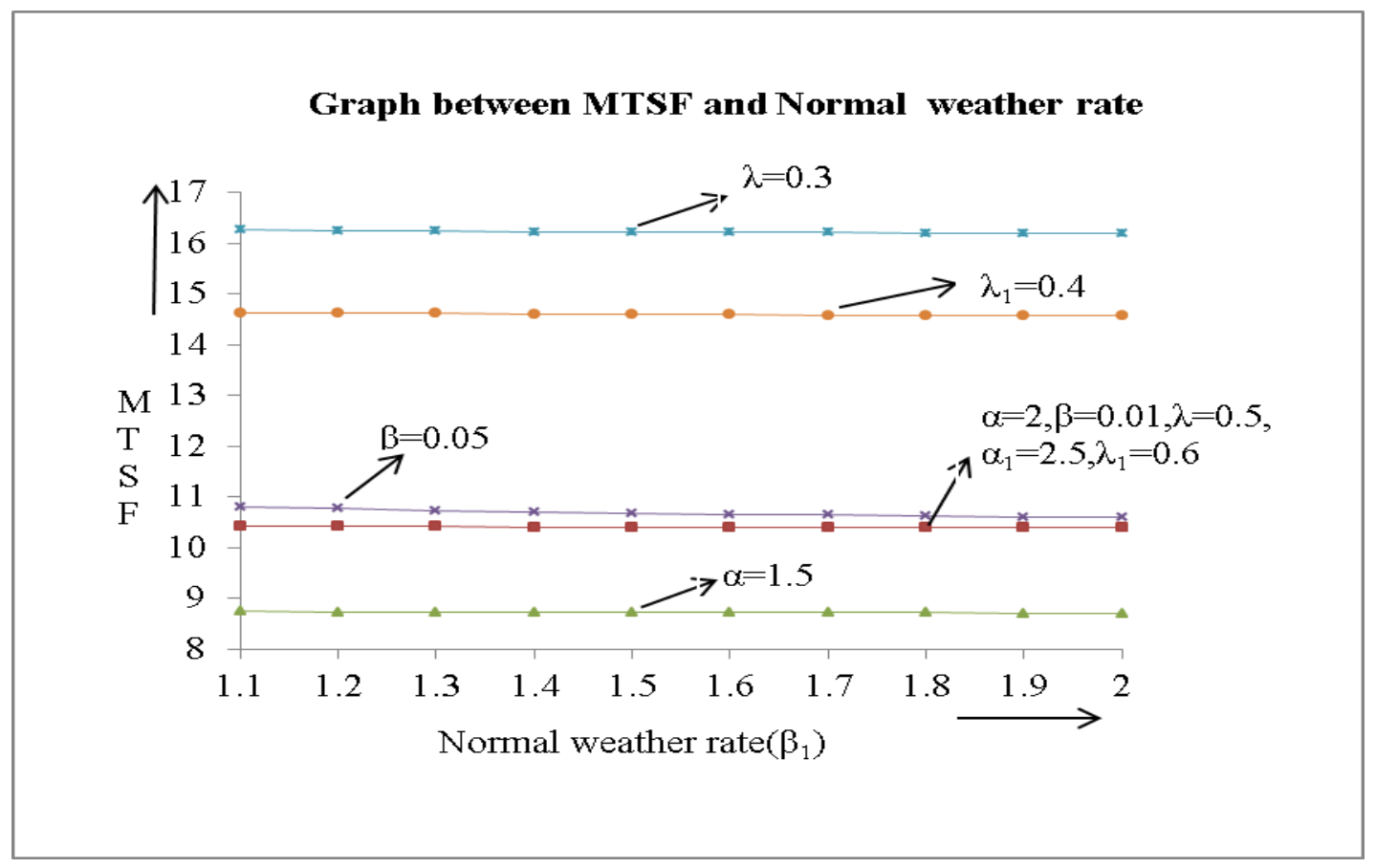

Fig : 2

Table: 1

\begin{tabular}{|c|c|c|c|c|c|}
\hline $\begin{array}{c}\text { Normal Weather } \\
\text { Rate }\left(\beta_{1}\right)\end{array}$ & $\begin{array}{c}\alpha=2, \beta=0.01, \\
\lambda=0.5 \lambda_{1}=0.6\end{array}$ & $\alpha=1.5$ & $\beta=0.05$ & $\lambda=0.3$ & $\lambda_{1}=0.4$ \\
\hline 1.1 & 10.42727 & 8.745455 & 10.80303 & 16.25758 & 14.63182 \\
\hline 1.2 & 10.41944 & 8.738889 & 10.76389 & 16.24537 & 14.62083 \\
\hline 1.3 & 10.41282 & 8.733333 & 10.73077 & 16.23504 & 14.61154 \\
\hline 1.4 & 10.40714 & 8.728571 & 10.70238 & 16.22619 & 14.60357 \\
\hline 1.5 & 10.40222 & 8.724444 & 10.67778 & 16.21852 & 14.59667 \\
\hline 1.6 & 10.39792 & 8.720833 & 10.65625 & 16.21181 & 14.59063 \\
\hline 1.7 & 10.39412 & 8.717647 & 10.63725 & 16.20588 & 14.58529 \\
\hline 1.8 & 10.39074 & 8.714815 & 10.62037 & 16.20062 & 14.58056 \\
\hline
\end{tabular}




\begin{tabular}{|c|c|c|c|c|c|}
\hline 1.9 & 10.38772 & 8.712281 & 10.60526 & 16.19591 & 14.57632 \\
\hline 2 & 10.385 & 8.71 & 10.59167 & 16.19167 & 14.5725 \\
\hline
\end{tabular}

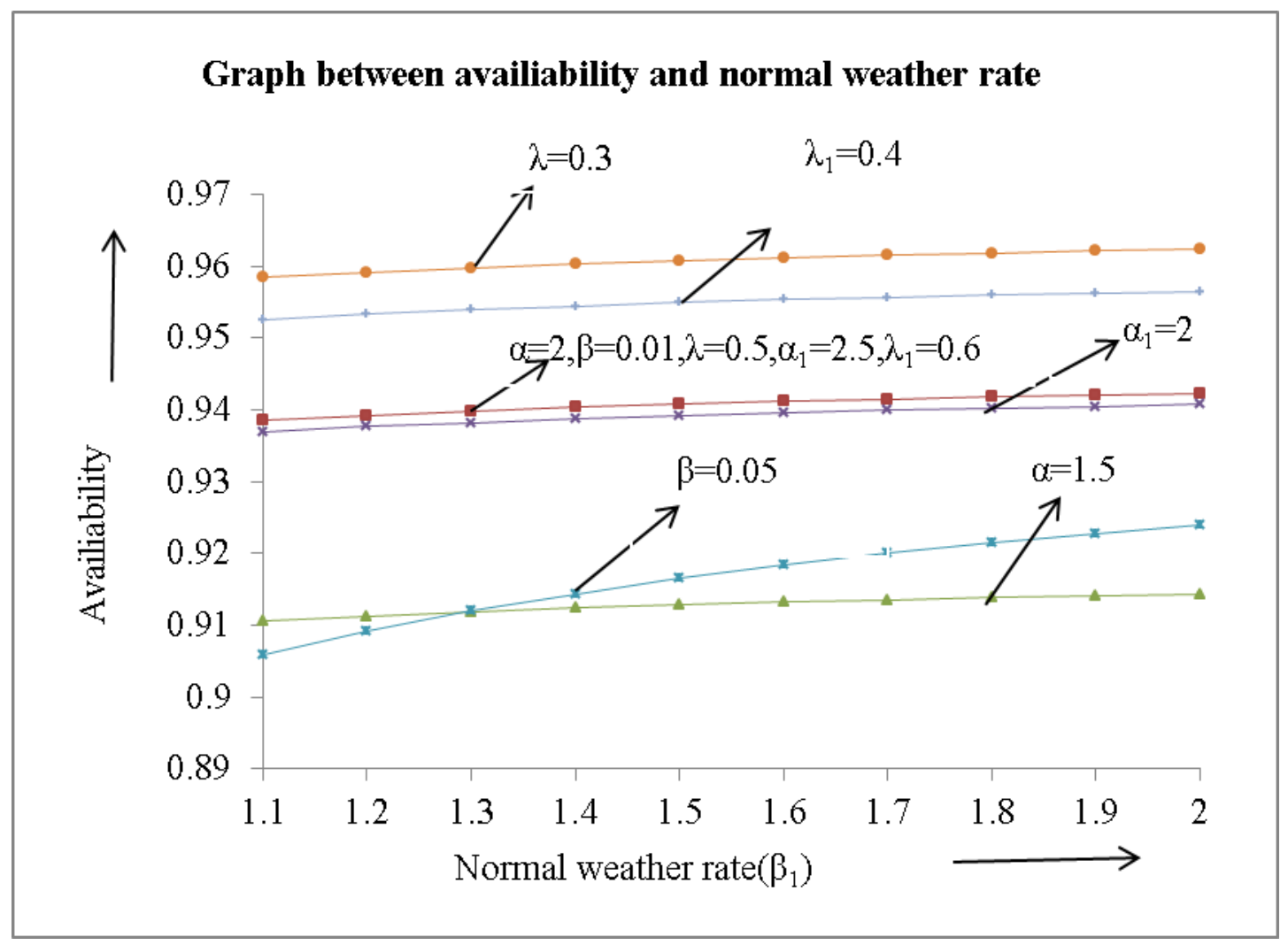

Fig.3

Table: 2

\begin{tabular}{|c|c|c|c|c|c|c|}
\hline $\begin{array}{c}\text { Normal weather } \\
\text { Rate }\left(\beta_{1}\right)\end{array}$ & $\begin{array}{c}\alpha=2, \beta=0.01, \lambda=0.5, \\
\alpha_{1}=2.5, \lambda_{1}=0.6\end{array}$ & $\alpha=1.5$ & $\alpha_{1}=2$ & $\beta=0.05$ & $\lambda=0.3$ & $\lambda_{1}=0.4$ \\
\hline 1.1 & 0.93853 & 0.91064 & 0.93694 & 0.90588 & 0.95848 & 0.95263 \\
\hline 1.2 & 0.93923 & 0.91132 & 0.93764 & 0.90918 & 0.9592 & 0.95335 \\
\hline 1.3 & 0.93983 & 0.9119 & 0.93824 & 0.91198 & 0.95981 & 0.95395 \\
\hline 1.4 & 0.94034 & 0.9124 & 0.93875 & 0.9144 & 0.96034 & 0.95447 \\
\hline 1.5 & 0.94079 & 0.91283 & 0.93919 & 0.91651 & 0.96079 & 0.95492 \\
\hline 1.6 & 0.94118 & 0.91321 & 0.93958 & 0.91836 & 0.96119 & 0.95532 \\
\hline 1.7 & 0.94152 & 0.91355 & 0.93993 & 0.92 & 0.96154 & 0.95567 \\
\hline 1.8 & 0.94183 & 0.91384 & 0.94023 & 0.92146 & 0.96185 & 0.95598 \\
\hline 1.9 & 0.9421 & 0.91411 & 0.9405 & 0.92278 & 0.96213 & 0.95626 \\
\hline 2 & 0.94235 & 0.91435 & 0.94075 & 0.92396 & 0.96238 & 0.95651 \\
\hline & & & & & & \\
\hline
\end{tabular}




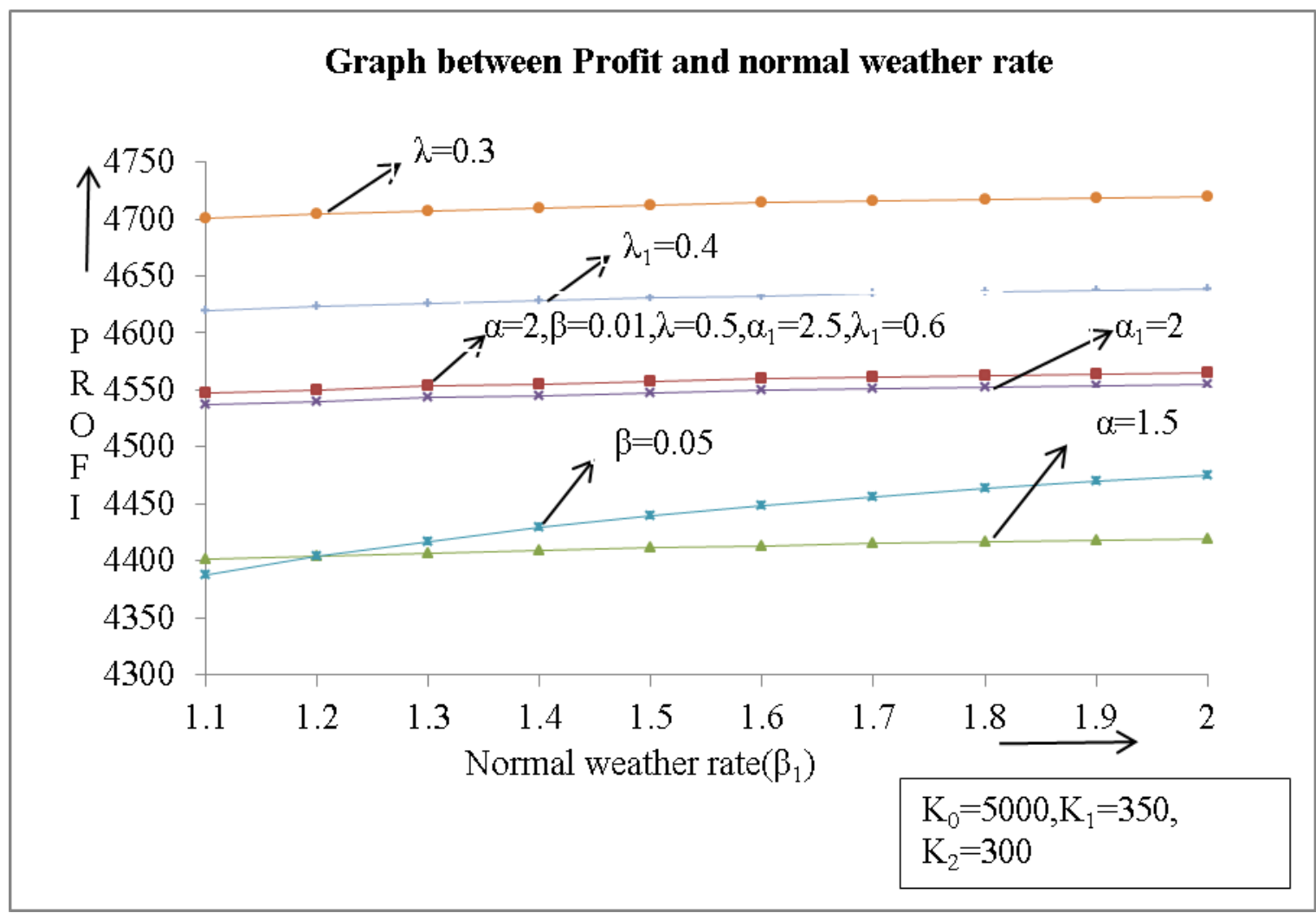

Fig: 4

Table:3

\begin{tabular}{|c|c|c|c|c|c|c|}
\hline $\begin{array}{c}\text { Normal } \\
\text { weather } \\
\text { rate }\left(\beta_{1}\right)\end{array}$ & $\begin{array}{c}\alpha=2, \beta=0.01 \lambda=0.5, \\
\alpha_{1}=2.5, \lambda_{1}=0.6, \\
\mathrm{~K}_{0}=5000, \mathrm{~K}_{1}=350, \\
\mathrm{~K}_{2}=300\end{array}$ & $\alpha=1.5$ & $\alpha_{1}=2$ & $\beta=0.05$ & $\lambda=0.3$ & $\lambda_{1}=0.4$ \\
\hline 1.1 & 4546.85 & 4401.32 & 4536.71 & 4387.82 & 4700.82 & 4619.86 \\
\hline 1.2 & 4550.27 & 4404.63 & 4540.12 & 4403.78 & 4704.35 & 4623.33 \\
\hline 1.3 & 4553.16 & 4407.43 & 4543.01 & 4417.37 & 4707.34 & 4626.27 \\
\hline 1.4 & 4555.64 & 4409.83 & 4545.49 & 4429.09 & 4709.91 & 4628.79 \\
\hline 1.5 & 4557.8 & 4411.92 & 4547.64 & 4439.29 & 4712.14 & 4630.98 \\
\hline 1.6 & 4559.69 & 4413.75 & 4549.52 & 4448.26 & 4714.09 & 4632.9 \\
\hline 1.7 & 4561.35 & 4415.36 & 4551.18 & 4456.21 & 4715.81 & 4634.59 \\
\hline 1.8 & 4562.84 & 4416.79 & 4552.66 & 4463.29 & 4717.34 & 4636.1 \\
\hline 1.9 & 4564.16 & 4418.08 & 4553.99 & 4469.65 & 4718.72 & 4637.45 \\
\hline 2 & 4565.36 & 4419.24 & 4555.18 & 4475.39 & 4719.95 & 4638.66 \\
\hline
\end{tabular}




\section{CONCLUSION}

The stochastic behavior of mean time to system failure (MTSF), availability and profit function has been observed on the basis of graphs obtained for a particular case as shown in figures 2, 3 and 4 respectively. From figure 2, it is analyzed that MTSF goes on decreasing with the increase of normal weather rate $\left(\beta_{1}\right)$ and failure rate $\left(\lambda\right.$ and $\left.\lambda_{1}\right)$. However, MTSF increases with increase of abnormal weather rate $(\beta)$ and repair rate $(\alpha)$ of the main unit. Figures 3 and 4 indicate that the values of availability and profit keep on increasing with the increase of normal weather rate $\left(\beta_{1}\right)$ and repair rates $\left(\alpha\right.$ and $\left.\alpha_{1}\right)$. But their values decline as and when abnormal weather rate $(\beta)$ and failure rates $(\lambda$ and $\left.\lambda_{1}\right)$ increase. Hence, the graph reveals that a system of nonidentical unit working under different weather conditions can be made more profitable by increasing repair rates of the main unit as well as by providing normal weather as much as possible for operation of the system.

\section{ACKNOWLEDGEMENT}

The author Ms. Savita Deswal is grateful to the DHE, Haryana and UGC, New Delhi for awarding Teacher Fellowship to carry out this research work.

\section{REFERENCES}

[1] Gopalan, M.N and Naidu, R.S (1984): Cost-Benefit Analysis of a One Server Two- Unit System Subject to Arbitrary Failure, Inspection and Repair. Reliability Engg., Vol. 8, pp. 11-22.

[2] Goel, L.R and Sharma, S.C (1989): Stochastic Analysis of Two -Unit Standby System with Two failure Modes and Slow Switch. Microelectron. Reliab., Vol. 29(4), pp. 493-498.

[3] Singh, S.K (1989): Profit evaluation of Two- Unit Cold Standby System with Random Appearance and Disappearance Time of the Service Facility. Microelectron. Reliab., Vol. 29, pp. 705-709.

[4] Mokkadis, G.S.; Elias, S.S. and Labib, S.W. (1989): On a Two Dissimilar Unit Standby System with Three Modes and Administrative Delay in Repair. Microelectron. Reliab., Vol. 29(4), pp. 511-515.

[5] Chander, S. (2005): Reliability Models with Priority for Operation and Repair with Arrival Time of Server. Pure and Applied Mathematika Sciences, Vol.LXI,No. 1-2,pp.9-22.

[6] Malik, S.C. amd Barak, M.S (2009): Reliability and Economic Analysis of a System Operating under Different Weather Conditions. Journal of Proc. Mat. Acad. Sci, India, Sect. A, Vol. 79, pt. II, pp205-213. 